\title{
Perfil da demanda em um serviço de Oftalmologia de atenção primária
}

\section{Demand profile in an Ophthalmologic primary care service}

Márcia Abelin Vargas ${ }^{1}$, Maria de Lourdes Veronese Rodrigues ${ }^{2}$

\section{Resumo}

Objetivo: Para melhorar os serviços de saúde é importante conhecer o perfil da demanda. Para isso, buscou-se:1) Identificar os principais motivos de procura por atendimento oftalmológico em Unidade de Atenção Primária; 2) Analisar as frequências dos diagnósticos,bem como os motivos de encaminhamento de pacientes para outros serviços de Oftalmologia. Métodos: Estudo descritivo e retrospectivo de 1.010 pacientes atendidos durante o período de 6 meses. Parâmetros: idade e sexo do paciente, motivo da consulta,diagnóstico (CID-10) e frequência de encaminhamentos. Os dados foram obtidos através da análise dos prontuários e das fichas de atendimento com os registros do CID-10. Alguns pacientes, de ambos os gêneros, apresentavam alterações anatômicofuncionais que foram agrupadas em mais de um grupo de diagnóstico. Resultados: Houve um predomínio do sexo feminino (61,5\%) e da faixa etária de 10 a 19 anos de idade $(16,7 \%)$, seguida do grupo de pacientes entre 40 a 49 anos de idade (16,5\%).O principal motivo da consulta foi a baixa de acuidade visual (37,4\%), seguida da revisão dos óculos $(19,0 \%)$. Também foram frequentes a queixa de cefaléia $(15,8 \%)$, prurido $(10,4 \%)$, ardência / irritação $(7,8 \%)$ e olho vermelho (7,2\%).Apresentaram exame normal, $8,1 \%$ dos pacientes e entre os exames anormais a maior frequência foi de erros refracionais $(70,0 \%)$. e presbiopia (31,9\%). Foram encaminhados $90(8,9 \%)$ pacientes. $\mathrm{O}$ principal motivo de encaminhamento foi a necessidade de cirurgia. Conclusão: $\mathrm{O}$ principal motivo da busca de um atendimento neste serviço básico de oftalmologia foi a baixa acuidade visual que na grande maioria das vezes foi ocasionada por erro refracional e/ou presbiopia. Poucos foram os casos não resolvidos no local do atendimento.

Descritores: Acuidade visual; Transtornos da visão; Oftalmopatias; Saúde ocular; Níveis de atenção à saúde; Atenção primária à saúde

\footnotetext{
'Doutora em Ciência Médicas, pelo Programa de Pós-graduação em Oftalmologia, Otorrinolaringologia e Cirurgia de Cabeça e Pescoço da Faculdade de Medicina de Ribeirão Preto da Universidade de São Paulo - USP - Ribeirão Preto (SP), Brasil.

${ }^{2}$ Professora Titular do Departamento de Oftalmologia, Otorrinolaringologia e Cirurgia de Cabeça e Pescoço da Faculdade de Medicina de Ribeirão Preto da Universidade de São Paulo - USP - Ribeirão Preto (SP), Brasil.
}

Trabalho realizado no Ambulatório de Oftalmologia da Unidade Mista de Saúde de Luís Antônio (SP), Brasil. 


\section{INTRODUÇÃO}

A procura pelo atendimento oftalmológico apresenta uma variabilidade de motivos,que depen dem de fatores biológicos, físicos, psíquicos, sociais e ambientais e em países em desenvolvimento, como o Brasil, há uma notável dificuldade em encontrar-se dados gerais de prevalência de distúrbios oculares nas diferentes faixas etárias.

A parcela da população formada por indivíduos portadores de distúrbios oculares ainda em fase moderada ou subclínica, de difícil identificação, assume proporção desconhecida. Supõe-se que tais indivíduos constituam a base do "iceberg" que,de forma figurada, representaria a perda da capacidade visual ${ }^{(1)}$. A cegueira e os distúrbios visuais apresentam ampla repercussão na sociedade, mais grave se considerado que $80 \%$ desses casos poderiam ter sido evitados ${ }^{(2)}$.

Em reunião internacional sobre atenção à saúde, realizada em Alma Ata, em 1978, se estabeleceu o seguinte conceito: "Atenção primária é assistência essencial à saúde, baseada em tecnologia e métodos científicos práticos e socialmente aceitáveis, deve ser universalmente acessível a todos os indivíduos e famílias, em sua comunidade e com sua participação, a um custo com o qual a comunidade possa arcar." Assim, os serviços podem ser oferecidos de forma contínua. Além disso, todo indivíduo que passar por um atendimento primário deve ter acesso a outros níveis de atenção à saúde, através de integração adequada, para maximização dos recursos disponíveis ${ }^{(3)}$.

Segundo a OPAS ${ }^{(4)}$, a oferta de serviços de atenção ocular deve ser planejada e organizada como parte de um sistema na qual se identifiquem os problemas, se criem planos para resolvê-los, se definam os recursos necessários para executar suas atividades e se programem as atividades e tarefas a serem realizadas, em diferentes níveis de complexidade. A hierarquização das ações de saúde e distribuição dos serviços é conhecida como atenção primária, secundária e terciária, sendo a primária conhecida como atenção básica de saúde. Dentro deste modelo considera-se que para contemplar os serviços de atenção ocular bastam dois níveis: nível de atenção básico e nível especializado. Os serviços básicos compreendem a identificação de fatores de risco, a promoção da saúde ocular, o diagnóstico precoce e, em algumas condições, os tratamentos simples; também inclui o controle periódico das pessoas sadias e enfermas e aspectos básicos da reabilitação.

O Município de Luís Antônio está localizado na região noroeste do Estado de São Paulo, com área total de 598 Km2. A população oficial, segundo o DATASUS em 2006, era de 8.134 habitantes $^{(2)}$, e a população fornecida através do Cadastramento Familiar, pelo Sistema de Informação de Atenção Básica - SIAB no ano de 2006, era de 10.153 habitantes ${ }^{(6)}$.

A Unidade Mista de Saúde de Luís Antônio é um estabelecimento de saúde público municipal que contém ambulatório de especialidades, pronto atendimento, urgência, emergências e leitos de observação. $\mathrm{O}$ ambulatório de especialidades funciona como referência local nas seguintes especialidades: ginecologia e obstetrícia, pediatria, clínica geral, ortopedia, cardiologia, oftalmologia, neurologia, urologia e saúde mental. Conta, também, com os seguintes serviços de apoio de baixa complexidade:laboratório de análises clínicas,serviços de Raios-X, ultrassonografia, E.C.G, ecocardiograma, farmácia,centro de odontologia, fisioterapia e equoterapia. A Unidade Mista de Saúde é a única alternativa de atendimento oftalmológico na cidade de Luís Antônio.

O consultório oftalmológico contém os equipamentos mínimos necessários para o atendimento básico oftalmológico: tabela de optotipos de Snellen, disposta à 6 metros da cadeira de exame do paciente, coluna pantográfica, foróptero, retinoscópio, oftalmoscópio direto e indireto e lâmpada de fenda. Não havia, na época em que a autora trabalhou no local, equipamento para a realização da tonometria ocular.

O direito a uma boa visão deve ser reconhecido como um importante componente de saúde pública, uma vez que possibilita um pleno desenvolvimento das potencialidades intelectuais e laborais humanas. Conhecer quais são as principais queixas e os principais problemas de saúde ocular de uma região pode ajudar a um melhor planejamento dos recursos públicos e a desenhar estratégias dirigidas à diminuição e controle das perdas visuais e da cegueira .

Apesar de existirem centenas de serviços de oftalmologia na rede pública brasileira, há uma escassez de informações na literatura científica nacional sobre o perfil ocular da população em geral. Procuramos, com esse estudo, colaborar com o arsenal literário oftalmológico.

\section{Métodos}

Foi realizado um estudo descritivo e retrospectivo dos prontuários médicos e das fichas de atendimento de 1.010 pacientes atendidos em consulta oftalmológica, realizada na Unidade Mista de Saúde de Luís Antônio- 
SP durante um período de 6 meses entre abril e setembro de 2006. Todos os pacientes haviam sido examinados pela autora (MAV).

Para a determinação do perfil dos pacientes atendidos foram extraídos dados referentes à faixa etária e sexo do paciente e motivos da consulta oftalmológica (queixas). Os diagnósticos foram categorizados de acordo com o capítulo VII da Classificação Estatística Internacional de Doenças e Problemas Relacionados à Saúde, Décima Revisão (CID-10) ${ }^{(7)}$ e o motivo de encaminhamento para outro serviço de oftalmologia, caso tenha ocorrido, foi levantado. Alguns pacientes, de ambos os gêneros, apresentavam alterações anatômico-funcionais que foram agrupadas em mais de um grupo de diagnóstico. Para o diagnóstico de ambliopia, consideramos acuidade visual menor que 20/40 ou diferença de duas linhas na tabela de Snellen entre os olhos. O diagnóstico de glaucoma foi obtido através da informação do paciente como sendo portador e em tratamento para o mesmo, e o de suspeita de glaucoma ou de glaucoma secundário à trauma, através da pressão bidigital e/ou escavação do nervo óptico suspeito. Foi registrado com o CID-10 H04.1 (Síndrome do olho seco)o paciente com queixas e que apresentava um tempo de ruptura do filme lacrimal, à instilação de 1 gota de colírio de fluoresceína, menor que 10 segundos. Também foi identificado, através da anamnese complementar, o paciente portador de diabete melito (DM) e/ou hipertensão arterial sistêmica (HAS).

Em todos os casos, os diagnósticos foram estabelecidos e registrados nas fichas de atendimento,com o CID-10, pela autora (MAV).

Após considerações sobre os aspectos éticos a pesquisa foi autorizada pela Diretora do Departamento de Saúde de Luís Antônio em 27 de setembro de 2006.

\section{$\underline{\text { RESULTADOS }}$}

A população atendida em consulta oftalmológica na Unidade Mista de Saúde de Luiz Antônio- SP, durante o período de abril a setembro de 2006 (6 meses), representou $12,4 \%$ da população oficial fornecida pelo DATASUS e 9,9\% da população fornecida pelo SIAB. Dos 1.010 pacientes atendidos, $61,5 \%(\mathrm{n}=621)$ pertenciam ao sexo feminino e $38,5 \%$ ao sexo masculino $(n=389)$. Em relação à idade, essa variou de zero (menos de 12 meses) a 94 anos, apresentando uma média de 34,8 anos (desvio padrão 20,9) e uma mediana 34 anos . A faixa etária prevalente entre o total de pacientes foi a de $10 \mathrm{a}$ 19 anos de idade (16,7\%), seguida pelo grupo de pacien- tes entre 40 a 49 anos de idade (16,5\%). A distribuição das faixas etárias de acordo com o gênero está demonstrada na tabela 1 .

Encontrou-se 31 motivos geradores de consulta oftalmológica. O principal motivo da consulta foi a baixa de acuidade visual $(37,4 \%)$, seguida da revisão dos óculos $(19,0 \%)$. Também foram frequentes a queixa de cefaléia $(15,8 \%)$, prurido $(10,4 \%)$, ardência / irritação $(7,8 \%)$ e olho vermelho $(7,2 \%)$.Considerando-se as faixas etárias prevalentes, a baixa acuidade visual $(37,9 \%)$ foi a queixa predominante seguida da cefaléia (29\%) na faixa etária dos 10 aos 19 anos e a baixa acuidade visual $(53,9 \%)$ seguida da revisão dos óculos $(16,8 \%)$ na faixa etária compreendida entre 40 e 49 anos de idade.

Houve uma prevalência de $15,1 \%$ de pacientes com história de HAS ( $\mathrm{n}=153)$, dos quais 99 (9,8\%) pacientes eram do sexo feminino e $54(5,3 \%)$ pacientes do sexo masculino. Em relação à presença de DM, 5,8\% dos pacientes sabiam ser portadores $(\mathrm{n}=59)$ sendo 40 $(3,9 \%)$ pacientes do sexo feminino e 19 (1,9\%) pacientes do sexo masculino.

Dez $(1 \%)$ pacientes apresentaram exame oftalmológico normal (seis do sexo feminino e quatro do sexo masculino). Se considerarmos o CID-10 H53.1 (Distúrbios visuais subjetivos) como sendo também exame oftalmológico normal, uma vez que não havia registro de um diagnóstico anatômico-funcional para a queixa, teremos um total de $82(8,1 \%)$ pacientes considerados como sem patologia ocular evidente (52 do sexo feminino e 30 do sexo masculino).

Dentre os exames anormais,os transtornos da refração (ametropias esféricas e cilíndricas isoladas ou combinadas) responderam por 70,0\% do CID-10 regis-

Tabela 1

\section{Distribuição das faixas etárias de acordo com o gênero}

\begin{tabular}{cccc}
\hline $\begin{array}{c}\text { Idade } \\
(\text { anos })\end{array}$ & $\begin{array}{c}\text { Sexo feminino } \\
\mathbf{N}(\%)\end{array}$ & $\begin{array}{c}\text { Sexo masculino } \\
\mathbf{N}(\%)\end{array}$ & $\begin{array}{c}\text { Total } \\
\mathbf{N}(\%)\end{array}$ \\
\hline $0-09$ & $71(11,4)$ & $62(15,9)$ & $133(13,2)$ \\
$10-19$ & $113(18,2)$ & $56(14,4)$ & $169(16,7)$ \\
$20-29$ & $104(16,7)$ & $50(12,9)$ & $154(15,2)$ \\
$30-39$ & $75(12,1)$ & $43(11,1)$ & $118(11,7)$ \\
$40-49$ & $106(17,1)$ & $61(15,7)$ & $167(16,5)$ \\
$50-59$ & $79(12,7)$ & $55(14,1)$ & $134(13,3)$ \\
$60-69$ & $44(7,1)$ & $28(7,2)$ & $72(7,1)$ \\
$70-79$ & $21(3,4)$ & $26(6,7)$ & $47(4,7)$ \\
$80-89$ & $08(1,3)$ & $06(1,5)$ & $14(1,4)$ \\
$>90$ & $0(0)$ & $02(0,5)$ & $02(0,2)$ \\
Total & $\mathbf{6 2 1}(\mathbf{1 0 0})$ & $\mathbf{3 8 9}(\mathbf{1 0 0})$ & $\mathbf{1 0 1 0}(\mathbf{1 0 0})$ \\
\hline
\end{tabular}


Tabela 2

Distribuição dos pacientes por gênero e grupo de diagnósticos

\begin{tabular}{lccc}
\hline Grupo de diagnósticos* & $\begin{array}{c}\text { Sexo feminino } \\
\text { N (\%) }\end{array}$ & $\begin{array}{c}\text { Sexo masculino } \\
\text { N(\%) }\end{array}$ & $\begin{array}{c}\text { Total } \\
\text { N(\%) }\end{array}$ \\
\hline Transtornos de Refração (ametropias esféricas) & $243(39,1)$ & $120(30,8)$ & $363(35,9)$ \\
Transtornos de Refração (astigmatismo) & $224(36,1)$ & $120(30,8)$ & $344(34,1)$ \\
Presbiopia & $196(31,6)$ & $126(32,4)$ & $322(31,9)$ \\
Transtornos da Pálpebra, do Aparelho Lacrimal e da Órbita & $97(15,6)$ & $70(18,0)$ & $167(16,5)$ \\
Transtornos da Conjuntiva & $88(14,1)$ & $79(20,3)$ & $167(16,5)$ \\
Distúrbios visuais subjetivos & $46(7,4)$ & $26(6,7)$ & $72(7,1)$ \\
Transtornos do Cristalino & $38(6,1)$ & $30(7,7)$ & $68(6,7)$ \\
Transtornos da Esclerótica, da Córnea, da Íris e do Corpo Ciliar & $11(1,8)$ & $29(7,4)$ & $40(4,0)$ \\
Transtornos da Coróide e Retina & $26(4,2)$ & $13(3,3)$ & $39(3,9)$ \\
Estrabismos & $15(2,4)$ & $06(1,5)$ & $21(2,1)$ \\
Ambliopia & $12(1,9)$ & $09(2,3)$ & $21(2,1)$ \\
Pseudofacia & $11(1,8)$ & $07(1,8)$ & $18(1,8)$ \\
Outros transtornos do Olho e Anexos & $06(1,0)$ & $07(1,8)$ & $13(1,3)$ \\
Glaucoma & $04(0,6)$ & $06(1,5)$ & $10(1,0)$ \\
Cegueira unilateral & $04(0,6)$ & $05(1,3)$ & $09(0,9)$ \\
Transtornos do Nervo Óptico e das Vias Ópticas & $02(0,3)$ & $03(0,8)$ & $05(0, \%)$ \\
Visão Subnormal uni ou bilateral & $02(0,3 \%)$ & $02(0,5 \%)$ & $04(0,4 \%)$ \\
\hline
\end{tabular}

${ }^{(*)}$ Alguns pacientes apresentavam mais de um diagnóstico

Tabela 3

Principais diagnósticos correlacionados com o gênero(feminino: $\mathrm{N}=389$; masculino: $\mathrm{N}=389$ ) e o número total de pacientes

\begin{tabular}{lccc}
\hline Diagnóstico (CID-10)* & $\begin{array}{c}\text { Sexo feminino } \\
\text { N (\%) }\end{array}$ & $\begin{array}{c}\text { Sexo masculino } \\
\text { N (\%) }\end{array}$ & $\begin{array}{c}\text { Total } \\
\text { N (\%) }\end{array}$ \\
\hline Blefarite & $47(7,6)$ & $24(6,2)$ & $71(7,0)$ \\
Pterígio & $34(5,5)$ & $34(8,7)$ & $68(6,7)$ \\
Catarata Senil Nuclear & $26(4,2)$ & $24(6,2)$ & $50(4,9)$ \\
Síndrome do Olho Seco & $25(4,0)$ & $17(4,4)$ & $42(4,2)$ \\
Conjuntivite Aguda Atópica & $18(2,9)$ & $18(4,6)$ & $36(3,6)$ \\
Conjuntivite Mucopurulenta & $15(2,4)$ & $12(3,1)$ & $27(2,7)$ \\
Ambliopia & $12(1,9)$ & $09(2,3)$ & $21(2,1)$ \\
Outras ceratites superficiais sem conjuntivite & $04(0,6)$ & $14(3,6)$ & $18(1,8)$ \\
Outras conjuntivites agudas & $08(1,3)$ & $08(2,0)$ & $16(1,6)$ \\
Degeneração da mácula e do pólo posterior & $07(1,1)$ & $04(1,0)$ & $11(1,1)$ \\
Cicatrizes coriorretinianas & $05(0,8)$ & $05(1,3)$ & $10(1,0)$ \\
Catarata Senil Incipiente & $07(1,1)$ & $03(0,8)$ & $10(1,0)$ \\
Suspeita de Glaucoma e Glaucoma & $04(0,6)$ & $06(1,5)$ & $10(1,0)$ \\
Hordéolo & $06(1,0)$ & $03(0,8)$ & $09(0,9)$ \\
Estrabismo convergente & $06(1,0)$ & $03(0,8)$ & $09(0,9)$ \\
\hline
\end{tabular}

${ }^{(*)}$ Alguns pacientes apresentavam mais de um diagnóstico

trado e a presbiopia (H52.4) com 31,9\%. Após, os cinco grupos mais encontrados foram, em ordem crescente de frequência: Transtornos da Pálpebra, do Aparelho Lacrimal e da Órbita (H00-H06); Transtornos da Conjuntiva (H10-H13); Transtornos do Cristalino (H25-H28); Transtornos da Esclerótica, da Córnea, da Íris e do Corpo Ciliar
(H15-H22) e os Transtornos da Coróide e da Retina (H30-H36) como representado na tabela 2.

Excetuando-se os transtornos de refração, a presbiopia e os distúrbios visuais subjetivos, os diagnósticos mais encontrados foram: blefarite, pterígio, catarata senil nuclear, síndrome do olho seco e conjuntivite 
aguda atópica. Ao agruparmos as conjuntivites, elas se tornaram responsáveis por 7,8\% dos atendimentos. Esses resultados estão referidos na tabela 3 .

Foram encaminhados para outro serviço de oftalmologia de nível terciário, 46 (7,4\%) pacientes do sexo feminino e 44 (11,3\%) do sexo masculino, de um total de $90(8,91 \%)$ pacientes. Os principais motivos de encaminhamento foram 28 casos de catarata $(2,8 \%), 13$ casos de glaucoma ou suspeita de glaucoma (1,3\%), 10 casos de pterígio (1\%) e cinco casos de estrabismo (0,5\%).

\section{DısCUSSÃo}

Houve um predomínio do sexo feminino, representando $61,5 \%$ do total de 1.010 pacientes, semelhante ao encontrado em outros estudos ${ }^{(8-10)}$. Isto pode ter sido ocasionado devido ao horário de atendimento oferecido ser o mesmo do horário comercial e sabe-se que ainda nos dias de hoje o sexo masculino é o responsável pelo provimento financeiro na maioria das famílias brasileiras, e portanto, teria menos oportunidades de ausentar-se de seu ofício para realizar a consulta.

A prevalência de hipertensão arterial sistêmica varia muito em diferentes estudos. Encontramos 15,1\% de pacientes que referiam ter diagnóstico clínico de HAS. Esse resultado está de acordo com os dados do Ministério da Saúde que estima que 16,9\% a $31 \%$ da população adulta brasileira seja hipertensa ${ }^{(11)}$. Em relação à presença de diabete melito, 5,8\% dos pacientes referiam ter o diagnóstico, o que ficou abaixo dos 7,6\% encontrado em estudo de âmbito nacional ${ }^{(12)}$ e dos $12,1 \%$ encontrados em estudo de prevalência de DM em uma cidade vizinha ${ }^{(13)}$. É importante frisar que não foi objetivo do presente estudo verificar a prevalência dessas duas importantes morbidades, uma vez que apenas foi verificado na história patológica pregressa, se o paciente referia ser hipertenso e /ou diabético.

Apresentaram exame normal 8,1\% dos pacientes atendidos e entre os exames anormais a maior prevalência foi de erros refrativos $(70 \%)$. A presbiopia representou $31,9 \%$ dos diagnósticos, o que não foi inesperado, uma vez que $43,2 \%$ dos atendimentos foram em pacientes com 40 anos de idade ou mais. Em estudos semelhantes a prevalência da presbiopia encontrou-se em torno de $33,6 \%$ a $37 \%{ }^{(8-10)}$. Após os Transtornos dos músculos oculares, do movimento binocular, da acomodação e da refração, os CID-10 mais encontrados foram os dos Transtornos da pálpebra, do aparelho lacrimal e da órbita e os Transtornos da conjuntiva, responsáveis por 33\% da casuística total. Em estudo sobre afecções oculares em crianças de zero a 15 anos de idade, Albuquerque e Alves $^{(14)}$ encontraram $13,4 \%$ de exame visual normal, os erros refrativos foram responsáveis por $65,1 \%$ dos diagnósticos e os Transtornos da conjuntiva e das pálpebras foi de $20 \%$.A prevalência de alterações no segmento anterior e anexos oculares demonstrou que as pálpebras e a conjuntiva representam as regiões oculares mais afetadas, sendo que a presença de pterígio correspondeu a $0,7 \%$ e a de blefarite foi de $3,5 \%$, porém o baixo número de adultos estudados justificou o achado ${ }^{(14)}$. Ao agruparmos as conjuntivites, elas se tornaram responsáveis por 7,8\% dos atendimentos na Unidade Mista de Saúde de Luís Antônio, seguidos pela blefarite (7\%), patologias que, na maioria das vezes, podem ser bem conduzidas pelo médico clínico generalista bem preparado ${ }^{(15)}$.

O pterígio afeta $8,2 \%$ da população rural adulta nigeriana ${ }^{(16)}$. Nós encontramos $6,7 \%$ de diagnóstico de pterígio, pois além de nossa amostra populacional ser representada por 70,1\% de indivíduos com 20 anos de idade ou mais, a cidade de Luís Antônio está localizada em uma região com alta exposição aos raios ultravioletas, agravado pelo fato da atividade econômica do município basear-se na agricultura, principalmente no plantio de cana-de-açúcar que ainda realiza o processo das "queimadas" antes da colheita, gerando ambiente seco e pulverulento, fatores de risco para o desenvolvimento dessa degeneração conjuntival ${ }^{(16-17)}$.

Na maioria dos países, $80 \%$ ou mais de todos ou casos de cegueira ou visão subnormal (VSN) encontramse na faixa etária acima dos 50 anos de idade. Esta faixa etária foi representada por $26,7 \%$ da população por nós estudada. Recente publicação sobre as causas de cegueira e VSN em nove regiões da América Latina apresentou uma proporção de pessoas com 50 anos ou mais, nas principais áreas urbanas, em torno de $20 \%$ e a catarata foi a principal causa de cegueira bilateral. De todas as causas encontradas de cegueira neste grande levantamento, $43 \%$ a $88 \%$ eram curáveis e $52 \%$ a $94 \%$ evitável (18). Nossa casuística encontrou $6,7 \%$ de Transtornos do cristalino, sendo que $4,9 \%$ correspondiam ao CID-10 H25.1 (catarata senil nuclear). Essa prevalência diminuída é, em parte, devida à proximidade do local da pesquisa a um grande serviço universitário público, o que permite fácil acesso ao tratamento cirúrgico desta patologia.

Dados sobre a epidemiologia da síndrome do olho seco são esparsos devido à variação na definição e nos diferentes parâmetros utilizados nas investigações, o que torna difícil a comparação entre os mesmos. Estudos apresentam prevalências maiores de síndrome do olho seco no sexo feminino (19-20), porém em nosso estudo elas foram similares $(4,0 \%$ para o sexo feminino e $4,4 \%$ para 
o sexo masculino). Existem testes específicos para o diagnóstico de olho seco, mas no presente trabalho foi feito apenas pelo tempo de quebra do filme lacrimal, o que seria um diagnóstico provável de ceratoconjuntivite seca de acordo com Paula e colaboradores ${ }^{(21)}$.

O principal motivo da consulta foi a baixa acuidade visual (37,4\%), seguida da revisão dos óculos $(19,0 \%)$, o que é esperado em se tratando de um serviço de oftalmologia ambulatorial. Semelhante achado $(45,3 \%)$ foi encontrado com relação ao motivo de procura por clientes em um serviço de referência secundária em oftalmologia na cidade de São Paulo ${ }^{(22)}$. Também foram frequentes a queixa de cefaléia $(15,8 \%)$, prurido $(10,4 \%)$, ardência / irritação $(7,8 \%)$ e olho vermelho $(7,2 \%)$, condizentes com os principais diagnósticos encontrados. Em 2007, um estudo sobre a medida da distribuição dos sintomas de desconforto visual em estudantes do ensino superior, que incluía baixa acuidade visual, cefaléia, irritabilidade, sensibilidade ocular entre outros,concluiu que há múltiplas etiologias para queixas oculares subjetivas ${ }^{(23)}$.

Foram encaminhados para outro serviço de oftalmologia de nível terciário 90 (8,9\%) pacientes. Os principais motivos de encaminhamento foram para resolução de patologias cirúrgicas: catarata $(2,8 \%)$, pterígio $(1 \%)$ e estrabismo $(0,5 \%)$.

Embora poucos casos foram encaminhados por glaucoma ou suspeita de glaucoma (1,3\%), é importante o rastreamento dessa patologia ocular que, se não tratada, leva sabidamente à cegueira irreversível. Sakata et al. ${ }^{(24)}$ em um estudo populacional no sul do Brasil, triaram 1.636 indivíduos com 40 anos de idade ou mais, referindo, por suspeita de glaucoma $17 \%$ da amostra, o que resultou em uma prevalência de diagnóstico de glaucoma em 3,4\% nesta população. Para a triagem, utilizaram lanterna, lâmpada de fenda, tonômetro de aplanação de Goldmann ${ }^{\circledR}$ e oftalmoscópio direto. Essa triagem poderia ser melhor realizada na Unidade Mista de Saúde de Luís Antônio se houvesse um investimento na aquisição do tonômetro, que também promoveria o acompanhamento terapêutico dos pacientes com diagnóstico de glaucoma, aumentando o poder resolutivo do nível básico do atendimento oftalmológico.

Com relação ao grau de resolutividade do Serviço de Oftalmologia de Luís Antônio obteve-se 91,1\% de resolução referente a essa demanda, valor este considerado muito bom, capacitando-o à resolução de problemas oftalmológicos que constituem a maior demanda na população e que não necessitam de procedimentos de maior complexidade.

\section{ConclusÃo}

O principal motivo da busca de um atendimento oftalmológico neste serviço básico de oftalmologia foi a baixa acuidade visual, que na grande maioria das vezes foi ocasionada por erro refracional e/ou presbiopia, seguida da revisão do óculos em uso. Os sintomas de cefaléia, prurido,ardência / irritação e olho vermelho podem ter a causa nos erros refracionais, ou nos principais diagnósticos encontrados (conjuntivites,blefarite, pterígio e síndrome do olho seco). Poucos foram os casos não resolvidos no local do atendimento, sendo a maioria de ordem cirúrgica. A falta de um tonômetro de aplanação de Goldmann ${ }^{\circledR}$ limitou a triagem e o acompanhamento do glaucoma e gerou encaminhamentos que poderiam ter resolutividade local se houvesse um investimento em equipamentos que não são tão onerosos.

\section{Agradecimentos}

As autoras agradecem a Dra. Sonia Mara Neves Ferri, Diretora do Departamento Municipal de Saúde de Luís Antônio, por ter autorizado a realização deste trabalho e a Professora Denise da Silva Fialho pelo auxílio na elaboração do resumo em inglês.

\section{Abstract}

Purpose: To improve health services it is necessary to know the demand profile. This study was carried out with the objectives of: to identify the main reasons to seek an ophthalmologic primary care service; and to analyze the frequency of the principal diagnoses, according to gender and age groups, as well as the causes of referring patients. Methods: Retrospective and descriptive study of 1,010 assisted patients during a period of 6 months. Parameters: age and sex of the patient, the reason of the visit, diagnosis (ICD-10) and frequency of referral. The data were obtained through the analysis of the medical records and data-recording sheets with the ICD-10 registration. Some patients (male and female) presented with different morphological and structural alterations, and were allocated in more than one diagnostic group. Results: There was a predominance of females $(61.5 \%)$ in the age group from 10 to 19 years of age (16.7\%) followed by the group of patients between 40 and 49 years of age (16.5\%). The main reason for the consultation was a low visual acuity (37.4\%), followed by the review of glasses (19.0\%). There were also frequent complaints of headache $(15.8 \%)$, pruritus $(10.4 \%)$, burning / irritation (7.8\%) and red eye $(7.2 \%)$. Among abnormal tests the largest prevalence was 
of refractive errors (70.0\%) and presbyopia (31.9\%). Ninety patients ( $8.9 \%$ ) were referred, mainly for surgical treatment. Conclusion: The main reason of the search of an Ophthalmology basic service was low visual acuity that in great majority of times was caused by refractive errors and/or presbyopia. Few were the cases which were not resolved at the service place.

Keywords: Visual acuity; Vision disorders; Eye diseases; Eye health; Health care levels; Primary health care

\section{RefERÊNCIAS}

1. Nizetic B. Perspectives in ophthalmology - a public health point of view. Can J Ophthamol. 8(3):311-6, 1973.

2. Resnikoff S. National prevention of blindness programmes and Vision 2020. J Com Eye Hlth. 2000;13(1):49-50.

3. Rodrigues M de LV. Prevenção de perdas visuais. Medicina (Ribeirão Preto).1997; 30 (1): 84-9.

4. Carazo V, Rico Velasco J, Beltranena F, Quesada T. Evaluating the conditions of efficiency. In: Carazo V, Rico Velasco J, Beltranena F, Quesada F. Guías para gerencia de servicios de atención ocular. Washington, DC: Organizacion Panamericana de la Salud; 1986.

5. Brasil. Ministério da Saúde - Cadernos de Informações de Saúde - DATASUS- [citado 2007 Set 5]. Disponível em: http:/ /tabnet.datasus.gov.br/tabdata/cadernos/sp.htm

6. Brasil. Ministério da Saúde - Sistema de Informação de Atenção Básica- SIAB -[citado 2007 Set 5]. Disponível em: http://www.datasus.gov.br/siab/siab.htm

7. Classificação Estatística Internacional de Doenças e Problemas Relacionados à Saúde, Décima Revisão. São Paulo, Brasil: Organização Mundial de Saúde,1993. [citado 2006 Mai 10]. Disponível em: url: http://www.datasus.gov.br/cid10/ webhelp/cid10.htm

8. Nwosu SN. Ocular problems of young adults in rural Nigeria. Int Ophthalmol. 1998;22(5):259-63.

9. Garcia CA, Pinheiro FI, Montenegro DA, Gomes AH, Oréfice F. Prevalence of biomicroscopic findings in the anterior segment and ocular adnexa among schoolchildren in Natal/Brazil. Arq Bras Oftalmol. 2005 Mar-Apr;68(2):167-70.

10. Verçosa IC, Maia EF. Perfil oftalmológico dos alunos do programa alfabetização solidária em quatro municípios do Ceará. Arq Bras Oftalmol. 2003;66(2):193-7.

11. Brasil. Ministério da Saúde. Secretaria de Vigilância em Saúde e Instituto Nacional do Câncer (INCA): Inquérito Domiciliar de Comportamentos de Risco de Morbidade Referida de Doenças e Agravos Não Transmissíveis: 17 capitais e Distrito Federal, 2002 - 2003 e 2004-2005. [citado 2007 Out 28]. Disponível em: http://tabnet.datasus.gov.br/ cgi/idb2006/d27b.htm

12. Malerbi DA, Franco LJ.Multicenter study of the prevalence of diabetes mellitus and impaired glucose tolerance in the urban Brazilian population aged 30-69 yr. The Brazilian Cooperative Group on the Study of Diabetes Prevalence. Diabetes Care. 1992;15(11):1509-16.
13. Torquato MT, Montenegro Júnior RM, Viana LA, DE Souza RA, Lanna CM, Lucas JC et al. Prevalence of diabetes mellitus and impaired glucose tolerance in the urban population aged 30-69 years in Ribeirão Preto (São Paulo), Brazil. São Paulo Méd J. 2003;121(6):224-30.

14. Albuquerque RC, Alves JGB. Afecções oculares prevalentes em crianças de baixa renda atendidas em um serviço oftalmológico na cidade do Recife-PE, Brasil. Arq Bras Oftalmol. 2003;66 (6):831-4.

15. PAsmanik S. Afecciones oculares más frecuentes. In: Meneghello J, Fanta E, Martínez AG, Blanco O. Pediatría práctica en diálogos. Argentina: Panamericana;2001.

16. Moran DJ; Hollows FC. Pterygium and ultraviolet radiation: a positive correlation. Br J Ophthalmol. 1984 May; 68 (5): $343-6$.

17. McCarty CA, Fu CL, Taylor HR. Epidemiology of pterygium in Victoria, Australia. Br J Ophthalmol. 2000; 84(3):289- 92.

18. Limburg H, Barria Von-Bischhoffshausen F, Gomez P, Silva JC, Foster A. Review of recent surveys on blindness and visual impairment in Latin America. Br J Ophthalmol. 92(3):315-9.

19. Mendes LE, Santos PM, Parente FSP, Gonçalves JOR, Belfort Junior R. Olho seco em pessoas com idade superior a 40 anos selecionadas em 3 cidades brasileiras. Arq Bras Oftalmol.1995;58(1):10-5.

20. Chalmers RL, Begley CG. Dryness symptoms among an unselected clinical population with and without contact lens wear. Cont Lens Anterior Eye. 2006;29(1):25-30.

21. Paula JS, Bonini-Filho MA, Schirmbeck T, Ferriani VPL, Rodrigues M de LV, Romão E. Estudo comparativo de testes diagnósticos para olho seco entre crianças saudáveis e portadoras de artrite reumatóide juvenil . Arq Bras Oftalmol. 2004; 67(5):769-72.

22. Gentil RM, Leal SMR, Scarpi MJ. Avaliação da resolutividade e da satisfação da clientela de um serviço de referência secundária em oftalmologia da Universidade Federal de São Paulo - UNIFESP - Arq Bras Oftalmol. 2003; 66 (2): 159-65.

23. Borsting E, Chase $\mathrm{CH}$, Ridder WH 3rd. Measuring visual discomfort in college students. Optom Vis Sci. 2007;84(8):745-51.

24. Sakata K, Sakata Lm, Sakata Vm, Santini C, Hopker Lm, Bernardes R et al. Prevalence of glaucoma in a South brazilian population: Projeto Glaucoma. Invest Ophthalmol Vis Sci. 2007;48(11):4974-9.

Endereço para correspondência:

Maria de Lourdes Veronese Rodrigues

Hospital das Clínicas - Campus

Dep. Oftalmologia, Otorrinolaringologia e Cirurgia

de Cabeça e Pescoço

Av. Bandeirantes, $\mathrm{n}^{\circ} 3900$

CEP 14049-900 - Ribeirão Preto (SP), Brasil

Fax: 16-3602-22426

e-mail: mdlvrodr@fmrp.usp.br 